\title{
A New Impedance Matching Method for an Ultra-Wide Band and Dual Circularly Polarised Feed
}

\author{
Sha Li ${ }^{1,4}$, Yi Hua Yan ${ }^{1}$, Wei Wang ${ }^{1}$, Zhi Jun Chen ${ }^{1}$, Dong Hao Liu ${ }^{1}$, Fu Shun Zhang ${ }^{2}$ and Natasha Jeffrey ${ }^{3}$ \\ ${ }^{1}$ Key Laboratory of Solar Activity, National Astronomical Observatories, Chinese Academy of Sciences, Beijing, 100012, China \\ ${ }^{2}$ Xidian University, Xi' an, 710071, China \\ ${ }^{3}$ School of Physics \& Astronomy, University of Glasgow, Glasgow, G12 8QQ, UK \\ ${ }^{4}$ Email: lisha1400@bao.ac.cn
}

(RECEIVED October 9, 2015; ACCEPTED October 19, 2016)

\begin{abstract}
In traditional antenna design, metal components are not placed in the central part of the antenna as they change the characteristics of near field radiation. However, we show that placing a metal ring in the centre of the strip lines, which connect the ends of folded high-frequency dipoles, does not damage the performance of the feed. Instead it significantly improves the voltage standing wave ratio of the feed whilst other performance indicators are not compromised. Thus, our findings show an excellent way of improving the wide band feed. Based on this foundation, a new circularly polarised feed for operation between 0.4 to $2 \mathrm{GHz}$ is introduced for the Chinese Spectral Radioheliograph in this paper. The issue of a feed impedance matching network is investigated. By optimising the impedance matching, the performance of the feed is enhanced with respect to the previous realisations of the Eleven feed. The simulation and experimental results show that the gain of the feed is about $10 \mathrm{dBi}$, and the VSWR is less than 2:1. In addition, the feed has a low axial ratio, fixed phase centre location, and constant beam width in the range of 0.4 to $2 \mathrm{GHz}$.
\end{abstract}

Keywords: Astronomical instrumentation - methods: analytical - methods and techniques

\section{INTRODUCTION}

Solar radio bursts are a product of sudden energy release during topological reorganisation of solar magnetic field (Kane et al. 2003) or magnetic reconnection. These phenomena occur across a wide range of frequencies from decimetric to centimetric wavelengths, as shown in Figure 1. Importantly, solar radio images provide the location of solar radio bursts. However, at present, solar radio imaging observations are only available at a few discrete frequencies, such as Gauribidanur Radioheliograph at $40-150 \mathrm{MHz}$ (Ebenezer et al. 2007), Nancay Radioheliograph (Kerdraon \& Delouis 1997) at $150-450 \mathrm{MHz}$, Siberian Solar Radio Telescope (Lesovoi et al. 2014) at $5.7 \mathrm{GHz}$, and Nobeyama Radioheliograph (Nakajima, Nishio, \& Enome 1994) at 17/34 GHz.

The Chinese Spectral Radioheliograph (CSRH, Wang et al. 2013) was developed to image solar radio bursts over a wide range of frequencies with high-frequency resolution. It provides a new tool for observing solar radio emissions including radio bursts from the primary energy release sites (Yan et al. 2008) of solar energetic events such as flares and coronal mass ejections (CMEs). The CSRH, as shown in Figure 2, consists of two interferometer arrays, CSRH-I and CSRH-II. The CSRH-I array is comprised of 40 reflector antennas with 4.5-m dishes and covers a frequency range from $0.4-2 \mathrm{GHz}$. The CSRH-II array is comprised of 60 reflector antennas with 2-m dishes and covers a frequency range from $2-15 \mathrm{GHz}(\mathrm{Li}$ et al. 2015b). All 100 antennas (Yan et al. 2012) are placed along three spiral arms with a maximum baseline of $3 \mathrm{~km}$ and surrounded by small hills (Liu et al. 2013). The CSRH will make full solar disk radio images at multiple frequency channels with a time resolution down to 3 ms (Acton 1996).

Dual circularly polarised feeds are designed to receive polarised solar radio signals. Since the Voltage Standing Wave Ratio (VSWR) is strongly dependent on the polarisation degree of the radio signal, it is desirable to design a feed with good characteristics. Circular polarisation measurements of solar radio emission reveal the relationships between the coronal magnetic field and energetic electrons, and a polarisation degree of $1 \%$ is detectable by radio heliographs. The VSWR must be as small as possible so that reflections in the antenna do not distort the very rapidly changing transient signals from the Sun. Previous ultra-wide band feeds, such as the quad-ridged horn (Bennett et al. 1976), corrugated horn 


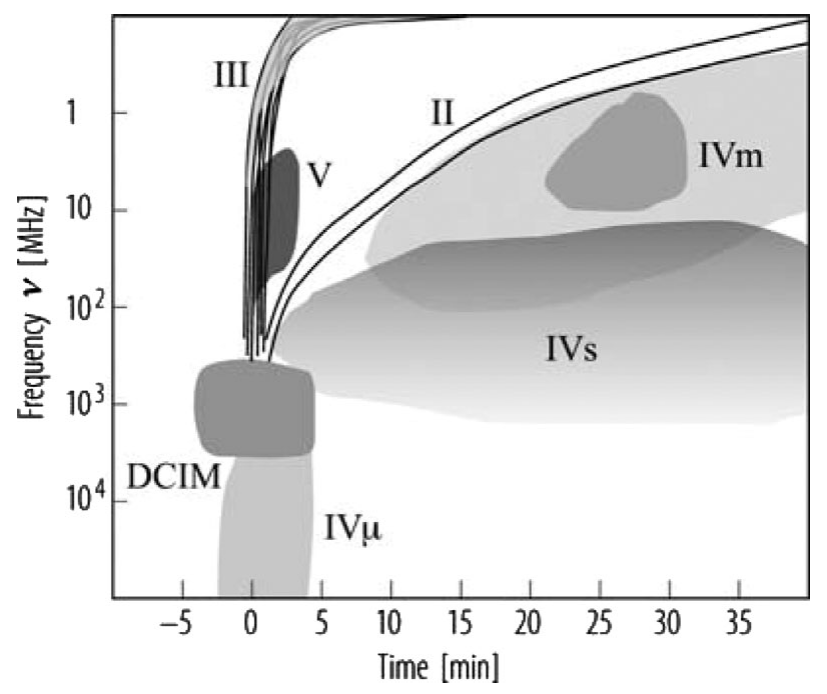

Figure 1. A schematic representation of the radio spectrum and different solar radio bursts during and after a large solar flare. The Roman numerals denote different types of metric bursts, with DCIM denoting decimetric emissions. Types IVm and IVs indicate moving and stationary Type IV bursts, respectively. Type IV $\mu$ indicates centimetre continuum due to gyrosynchrotron emission. It may continue beyond $100 \mathrm{GHz}$. Figure and caption taken from Benz (2009).

(Kraus \& Marhefka 2002), and sinuous antenna (Aghdam, Farajj-Dana, \& Rashed-Mohassel 2005), are not suitable for the observation of fast changing radio frequency (RF) signals. The Eleven feed was first developed at Chalmers University in 2005. In Olsson, Kildal, \& Weinreb (2006), the reflection coefficient of the original Eleven feed was reported as -5 $\mathrm{dB}$ in the range $0.15-1.5 \mathrm{GHz}$, which can be used to observe objects with slowly changing signals. However, it is not suitable for observing the Sun since solar radio bursts may vary rapidly at millisecond time scales. In Yang et al. (2011), a new system design for reflector antennas was introduced using a cryogenic $2-13 \mathrm{GHz}$ Eleven feed. The reflection coefficient was below $-8 \mathrm{~dB}$ in the ultra-wide band width. In Yin et al. (2013), the performance of the circular Eleven feed shows that the measured reflection coefficient is below $-8 \mathrm{~dB}$ in the $2-14 \mathrm{GHz}$ range. However, this performance is still not satisfactory for the requirements of solar radio observations with fast changing signals. Also, in Yang et al. (2015) and Yang (2014), a new type of Eleven feed with high sensitivity over $0.35-1.05 \mathrm{GHz}$ range was introduced for the Square Kilometer Array (SKA) project band 1, improving the reflection coefficient below $-12 \mathrm{~dB}$. However, in the low and high-frequency bands, the reflection coefficients vary in the range of $-12 \mathrm{~dB}$ to $-4 \mathrm{~dB}$. Further, the isolation between two orthogonal ports is larger than $50 \mathrm{~dB}$ and the aperture efficiency is above $70 \%$ in the high part of the band. To facilitate the requirement of the CSRH observing the Sun ( $\mathrm{Li}$ et al. 2014), we design another circularly polarised feed. It refers to the Eleven feed of Chalmers University (Olsson et al. 2006) by improving its VSWR (Kildal 2000).

The difference between these feeds lies in the impedance matching and the method of synthesis of dual circularly polarised signals. In the feed testing stage, we found that placing a metal ring in the centre of the microstrip line can improve the VSWR significantly, achieving good impedance matching. Our feed design uses a state-of-the-art principle, the placement of a metal ring (Kim et al. 2008), instead of a balun (Zhang, Peng, \& Xin 2008) . We will show, using systematic simulations, the efficiency and effectiveness of this new method. Our method also improves upon the detection of dual circularly polarised signals. In our method, there are four log-periodic arrays in the feed, and each pair of opposite log-periodic arrays are connected by the microstrip line at the foot of the arrays, i.e. at the high-frequency ends. The

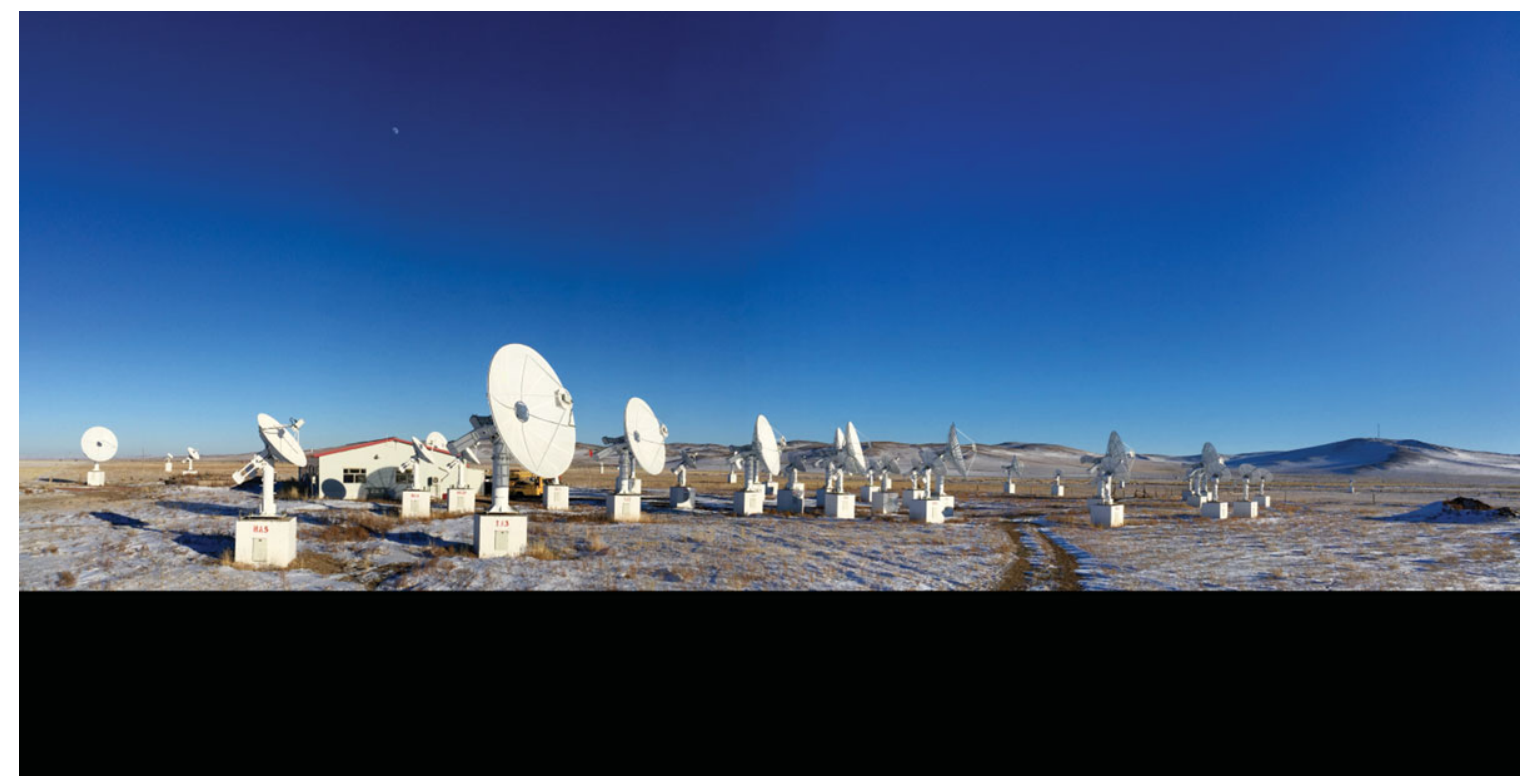

Figure 2. A picture of the CSRH. 


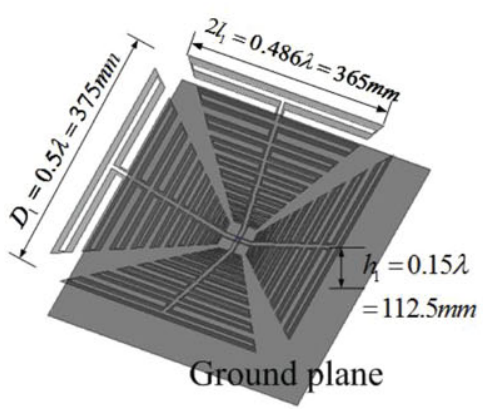

(a)

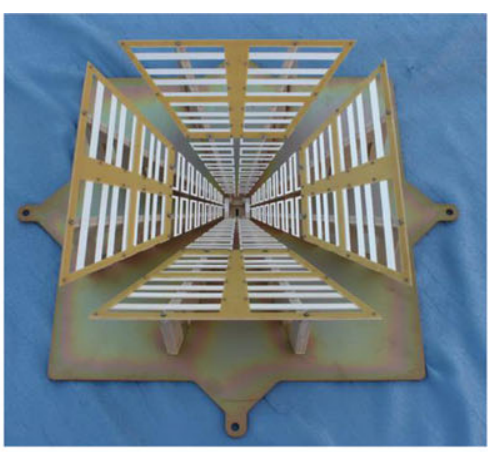

(b)

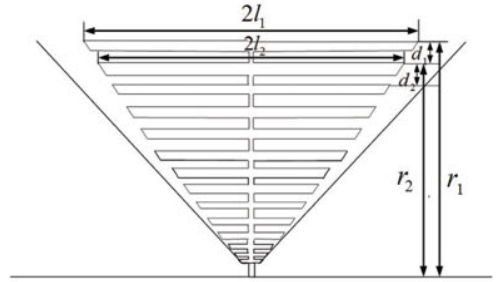

(c)

Figure 3. The geometries of the proposed design. (a) Top view of feed. (b) The manufactured feed. (c) Side view of the feed.

coaxial inner core and cable skin are linked with this microstrip line separately, so there are two output ends of the feed in total, which decreases the interference of the cable lines. In Yang et al. (2011), there are eight outputs at the ends of the log-periodic arrays. These outputs are combined with two $180^{\circ}$ hybrids (balun), four loads, and one $3 \mathrm{~dB}$ power combiner to form a feeding network. Such a feeding network would consume more energy and result in signal loss due to the complex structure, compared with Yang et al. (2015). The microstrip line we use for both vertical and horizontal polarisations is straight and does not have step transformer-type lines. We use straight microstrip lines to form one polarisation and the main idea is to remove the mutual coupling by using two centre-bridge geometries. For the vertical polarisation, a coaxial cable is fed with the inner conductors connected to one strip line, and the outer conductors connected to another strip line which is also connected to the main ground plane. The measurement of horizontal polarisation uses the same setup.

This paper mainly studies the issue of impedance matching and gives the performance of the CSRH-I antenna. The feed of CSRH-II is introduced in another paper. This paper is organised as follows: Section 1 shows the software simulations of a metal ring placed in the microstrip line of our feed, Section 3 presents the software simulations and actual measurements of our feed, and Section 4 is the conclusion.

\section{THE SIMULATION OF IMPEDANCE MATCHING USING A METAL RING}

The goal of this feed design is to minimise the VSWR of the output port and obtain good radiation patterns over the frequency band of the CSRH. To achieve good performance, it is necessary to optimise impedance matching. For wideangle scanning array antennas, the magnitude of the reflection changes substantially with scan angle and wave polarisation. Conventional matching techniques in the individual element of feed lines cannot compensate for these changes. There is a method of wide-angle impedance matching (WAIM) (Sikina 2010) which utilises a thin, high-k dielectric sheet spaced in front of the array. In Deng et al. (2006), stepped-impedance resonators play important roles for the matching circuits. In our application, during the testing stage of our feed design, a metal ring was placed at the centre of the microstrip line resulting in good impedance matching and therefore an improved VSWR. To explain and verify such an operation, a series of simulations using High Frequency Structure Simulator (HFSS) software is implemented in this section.

The simulated model and measured model are shown in Figure 3. Figure 3(a) shows the simulated model and the geometry and dimensions of the Eleven feed. The prototype picture of the CSRH feed is shown in Figure 3(b). The side view of the feed and the central part of Figure 3 is shown in detail in Figure 3(c). In Figure 3(c), the parallel solid lines represent two microstrip lines that connect the two opposite dipole log-periodic arrays. The two microstrip lines are connected with the ends of a coaxial line to output two signals. These two signals are combined after a $90^{\circ}$ phase shift. $l_{i}$ represents the length of half a wave dipole $i, d$ represents the separation between two adjacent dipoles, and $r$ is the distance from the dipoles to the vertex.

In our simulation, for computing efficiency, a feed consists of a pair of oppositely located log-periodic dipole arrays. The simulated model of the feed with a metal ring is shown in Figure 4. It shows the side view of the central part, where $\mathrm{dr} 1$ and $\mathrm{dr} 2$ represent outer and inner diameters of the symmetrical circle, respectively. The centre is connected to a balanced microstrip line, and a metal ring was placed above the microstrip line.

Figure 4 shows the geometry of the simulated model with the metal ring geometry. It is a model of a pair of log-periodic arrays for one polarisation. The simulated results were calculated by the software HFSS. We did not find a suitable way of fixing the metal ring to the microstrip lines for the real object feed, so we use four 470 impedances instead of the metal ring geometry.

The simulated results verify the advantage of adding a metal ring above the microstrip line. Figure 5 shows the simulated results with variable sizes and positions of the metal ring in the feed. The poorest performance of this feed occurs 


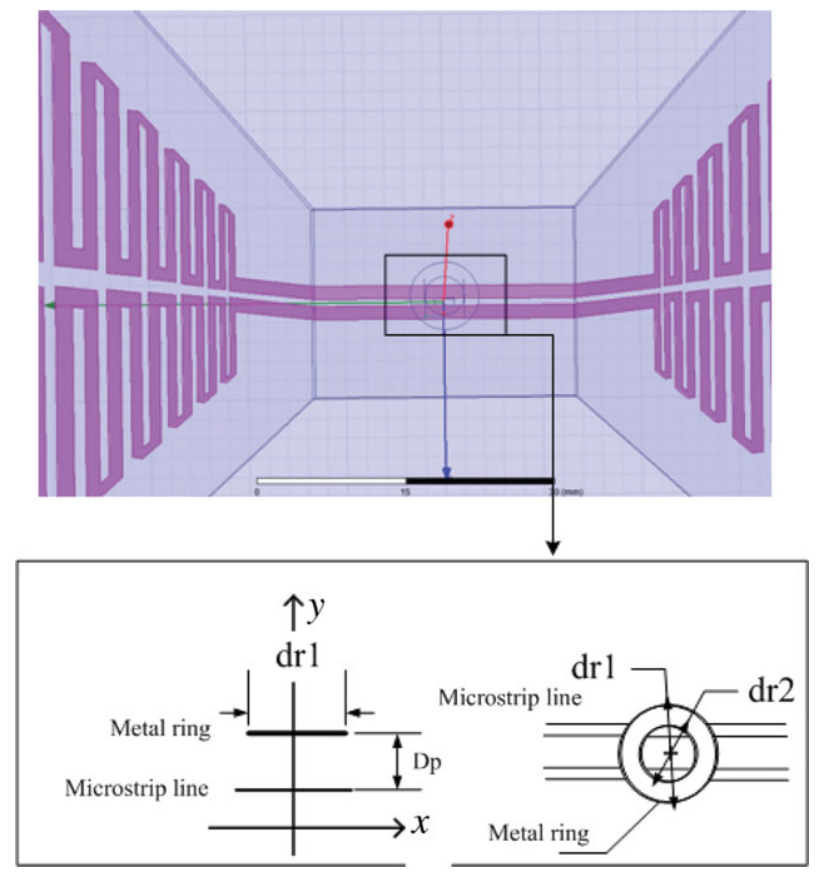

Figure 4. The simulated model with a metal ring (top). The side view and the top view of the central part of the feed (bottom).

when the metal ring is excluded. However, simulating with a metal ring improves the VSWR, whilst the efficiency and radiation patterns are almost the same in different circumstances. The average radiation efficiency is about 0.96 , with only a few circumstances showing poor efficiency. Figure 6 shows the simulated radiation patterns at $0.4,1.2,1.6$, and $2.0 \mathrm{GHz}$, for different models. We can see that the radiation patterns appear almost the same in different models and there-

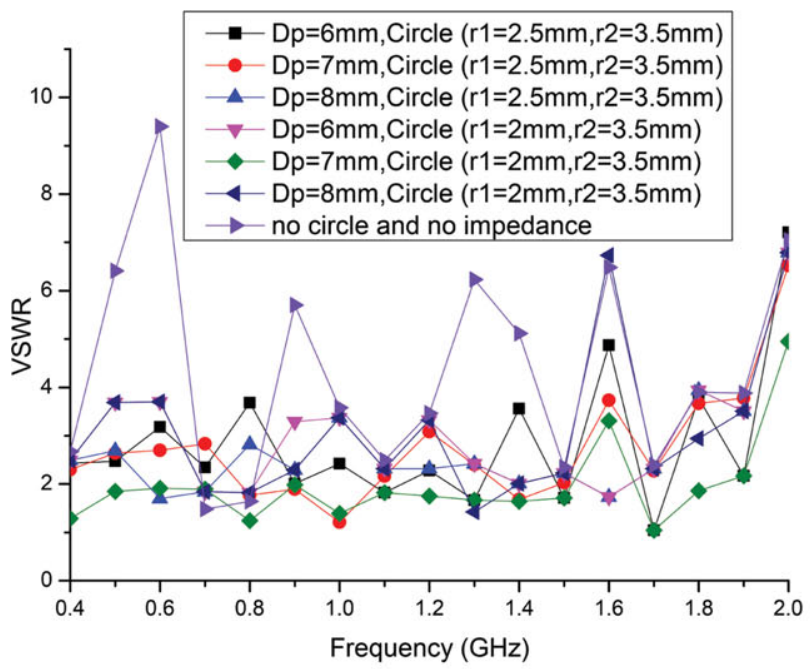

(a) fore, the method of adding a metal ring to improve impedance matching is applicable.

The signal from the feed is imported into the following analogue receiver. The feed and the analogue receiver are connected by a transmission line. To achieve impedance matching, the resistance of the combination of the metal ring and the feed should be equal to the impedance of the transmission line; meanwhile, the reactance should be very small so that the imaginary part of the impedance is near to zero.

\section{THE PERFORMANCE OF THE DESIGNED FEED WITH THE PROPOSED IMPEDANCE MATCHING}

The ideal feeding network used in the Eleven feed (Yang et al. 2009) consists of two ideal $180^{\circ}$ hybrids (balun) and one ideal $3 \mathrm{~dB}$ power combiner, which includes a central puck and a real eight-port circuit board. In our CSRH feed, two log-periodic arrays are placed face-to-face to form one polarisation array receiving one polarised signal. These two polarisation arrays are connected by two microstrip lines. The coaxial inner core and cable skin are linked with these two microstrip lines separately and thus a voltage difference between these two dipoles is achieved.

The relationships of the log-periodic arrays are as follows:

$$
\begin{gathered}
\frac{l_{n}}{l_{n-1}}=\frac{r_{n}}{r_{n-1}}=\frac{d_{n}}{d_{n-1}}=\tau, \\
d_{n}=(1-\tau) * r_{n}, \\
\theta=\tan ^{-1}\left\{\frac{l(1-\tau)}{d_{n} / \lambda_{n}}\right\} .
\end{gathered}
$$

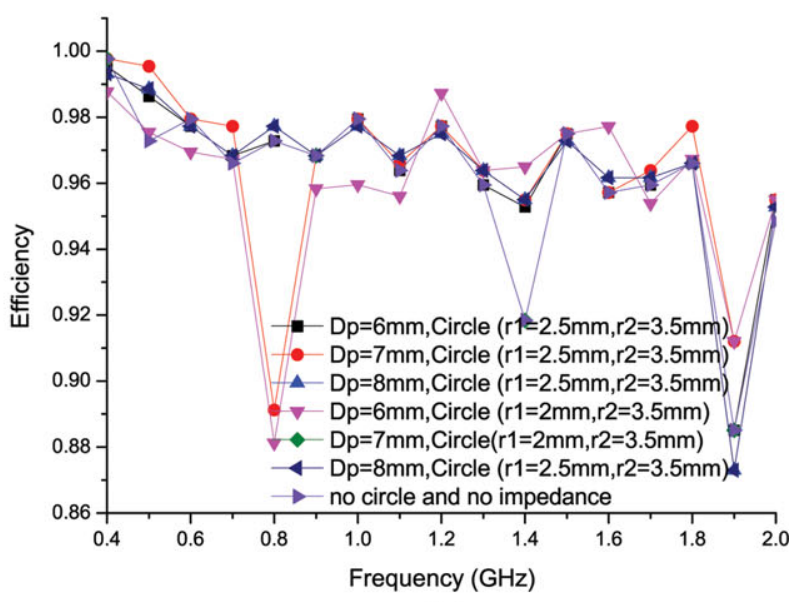

(b)

Figure 5. The simulated results [(a) VSWR and (b) Antenna efficiency] using different distances Dp and ring size (inner diameter is dr1 and outer diameter is dr2). Dp represents the distance between the ring and the dielectric slab. Each figure includes seven different circumstances with different colours corresponding to different parameters. (a) The simulated VSWR. (b) Antenna efficiency. 

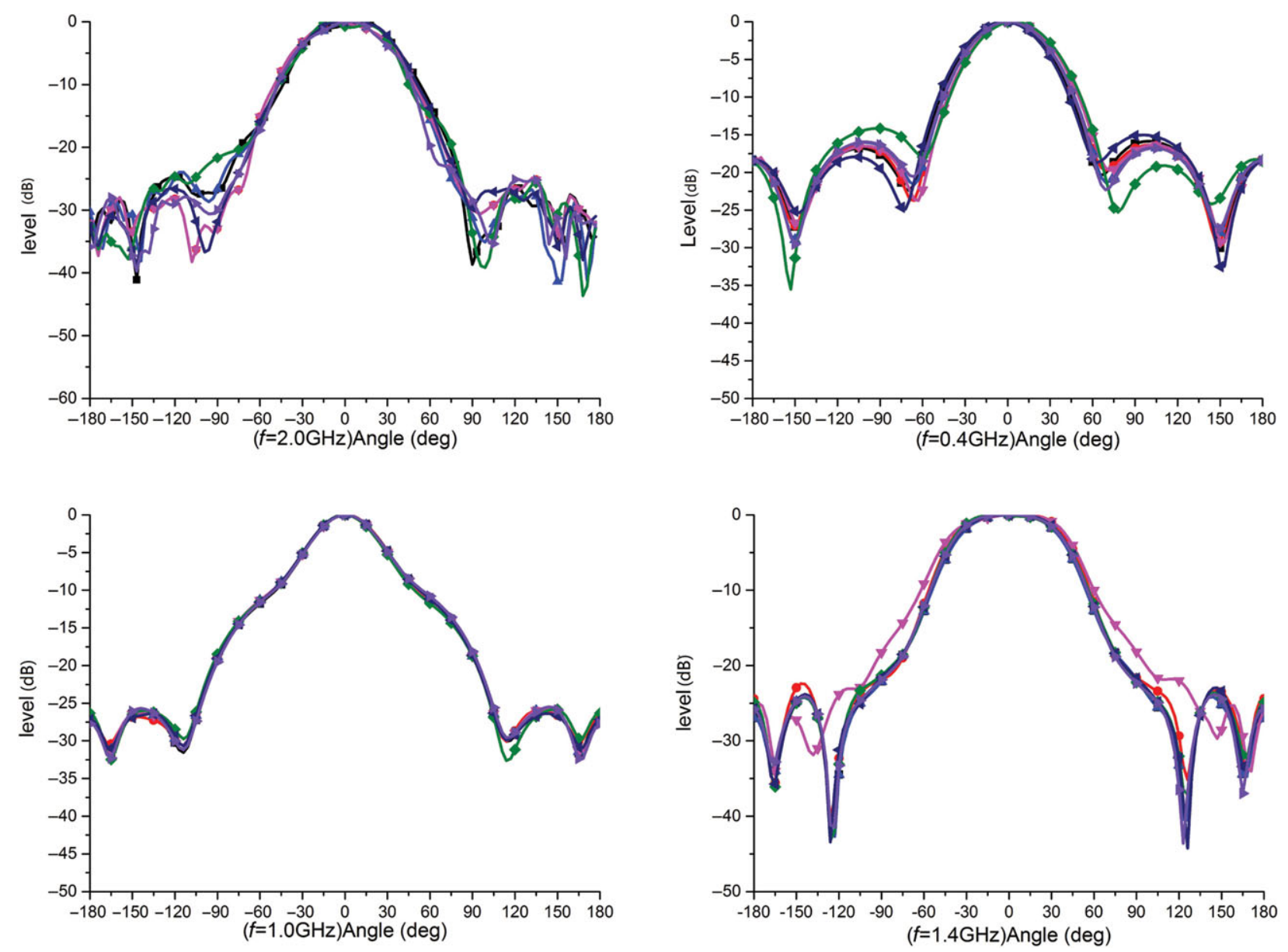

Figure 6. The simulated radiation patterns at different frequencies (the different coloured lines correspond to different circumstances, as shown in Figure 5).

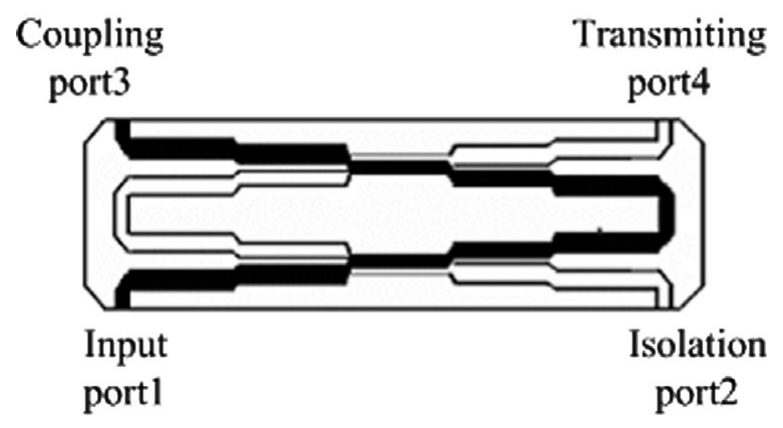

Figure 7. The schematic drawing of a $90^{\circ}$ hybrid.

Two adjacent dipoles above the ground plane generate bore-sight radiation patterns. In order to get circularly polarised signals, a wideband $3 \mathrm{~dB}$ hybrid (Cristal \& Young $1965)$ is synthesised using multiple symmetrical parallel offset strip lines (Shelton 1966). Figure 7 shows the schematic drawing of the $90^{\circ}$ hybrid. We selected a ripple value of 0.25 $\mathrm{dB}$ and the number of strip lines is 5 . This number sets the size of each microstrip line. This is simulated using Advance Design System (ADS) software (Qing \& Chia 1999). Next,

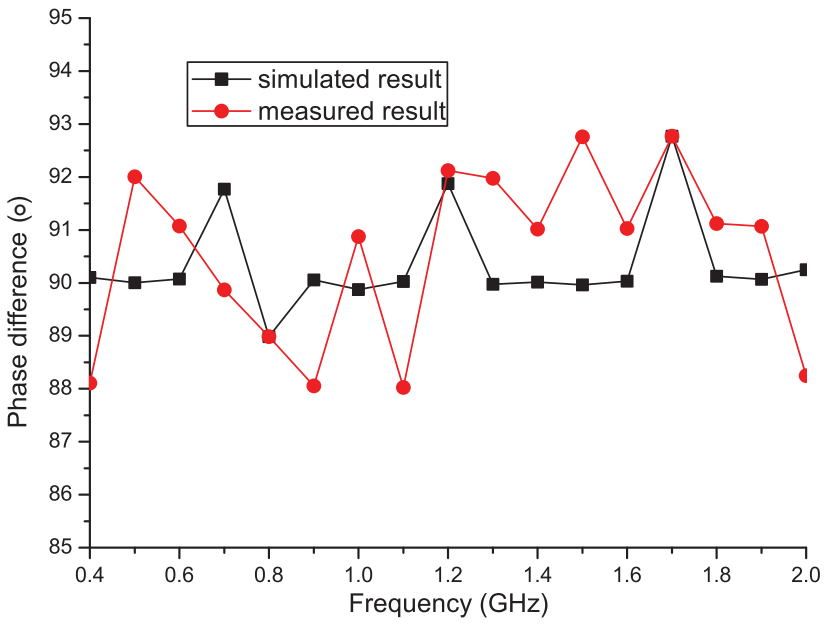

Figure 8. The simulated and measured results of phase difference for the hybrid output.

we manufactured the hybrid and tested it with a vector network analyser. Figure 8 shows the phase distance of the two outputs from the hybrid and indicates the phase difference of the hybrid output. The phase difference in the wide frequency 


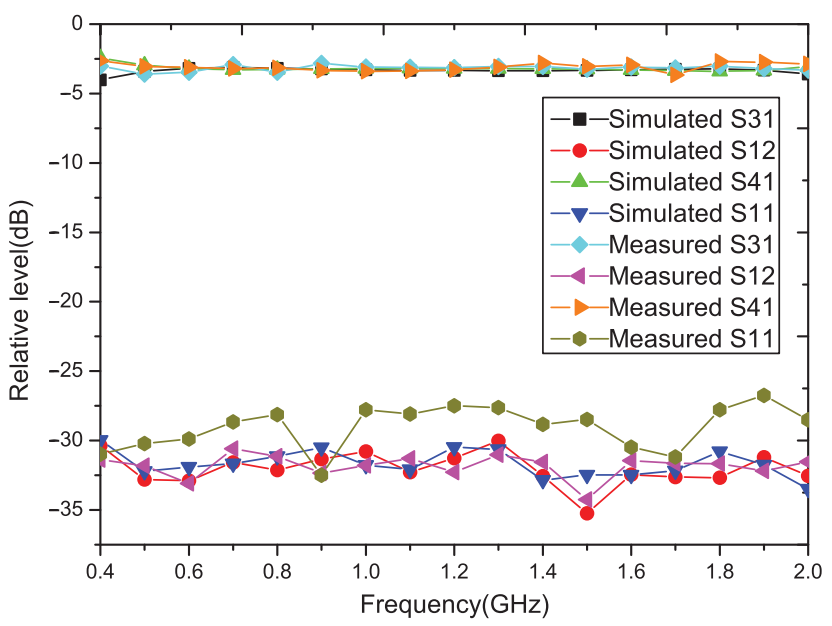

Figure 9. The results of the $S$ parameter characteristics.

band is almost $90^{\circ}$ with no more than a $3^{\circ}$ variation. Figure 9 shows the $S$ parameters of the hybrid, where the measured and simulated transmission coefficients $S_{31}$ and $S_{41}$ are almost -3 $\mathrm{dB}$ and the measured and simulated $S_{11}$ and $S_{21}$ are less than $-27 \mathrm{~dB}$. It indicates that the signal transmission efficiency is good and signal loss is very small. The results show consistency between the simulations and the actual measurements in Figures 8 and 9.

The far-field pattern measurements of the feed were carried out in an anechoic chamber (Yang \& Kildal 2000). The distance between the source and the feed under test was about $15 \mathrm{~m}$. The radiation patterns (Richard \& Jasik 1984) of the proposed feed in the $\mathrm{E}$ and $\mathrm{H}$ planes are listed in Figure 10 for $0.4,1.2$, and $2.0 \mathrm{GHz}$. It can be seen that the co-polar patterns have a nearly constant beam width at these frequencies. The axial ratio was measured in this chamber too. In general, Figure 11 shows that the axial ratio band is below $2.5 \mathrm{~dB}$, over the whole band. Figure 12 shows the measured feed gains, varying in the range of 8.9-11.2 dB across the whole operating frequency bands. Again, it can be seen that the measured results agree well with the simulated results.

The VSWR of the CSRH-I feed was simulated using Ansoft HFSS (Xie et al. 2007) and measured using an Agilent E8363B PNA network analyser, as shown in Figure 13. Figure 13 shows that the measured results agree reasonably

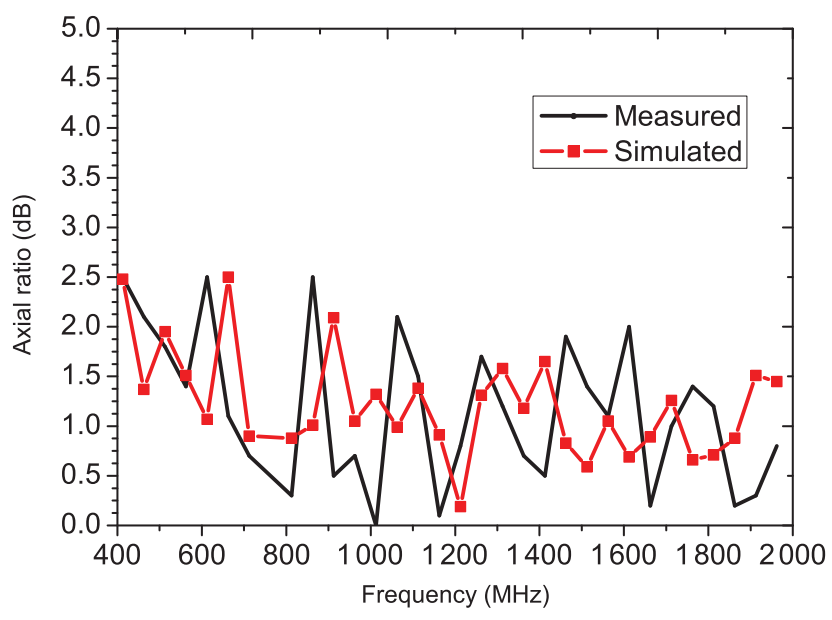

Figure 11. The simulated and measured axial ratio.

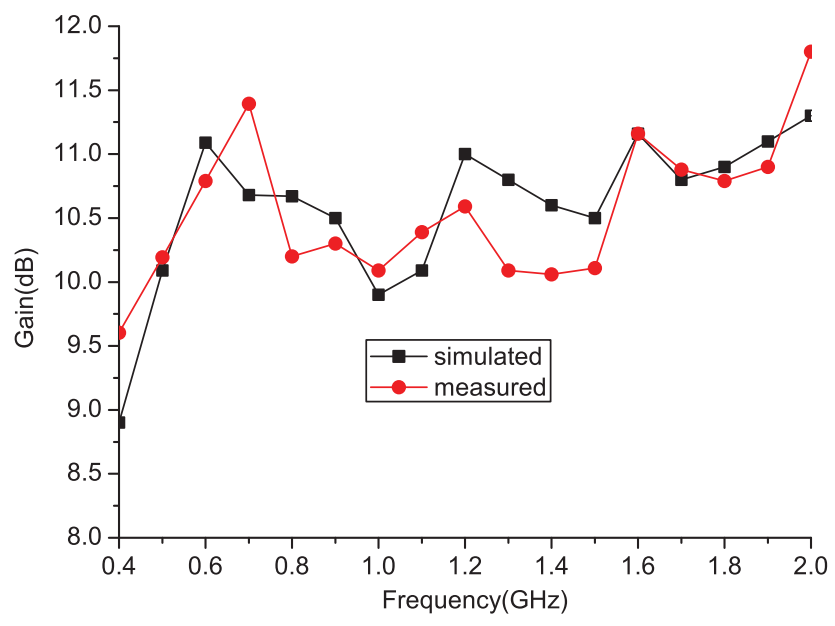

Figure 12. The simulated and measured peak gain of the feed.

well with the simulated ones, with values below 2 from 0.4 to $2 \mathrm{GHz}$.

CSRH uses a primarily focussed reflector antenna and the focal ratio (F/D) is 0.4 . Figure 14 shows the simulated 3D radiation patterns at $0.4 \mathrm{GHz}$. The radiation pattern is symmetrical and its illumination angle of the $10 \mathrm{~dB}$ beam width is about $135^{\circ}$. The simulated total gain is about $27.7 \mathrm{~dB}$. The
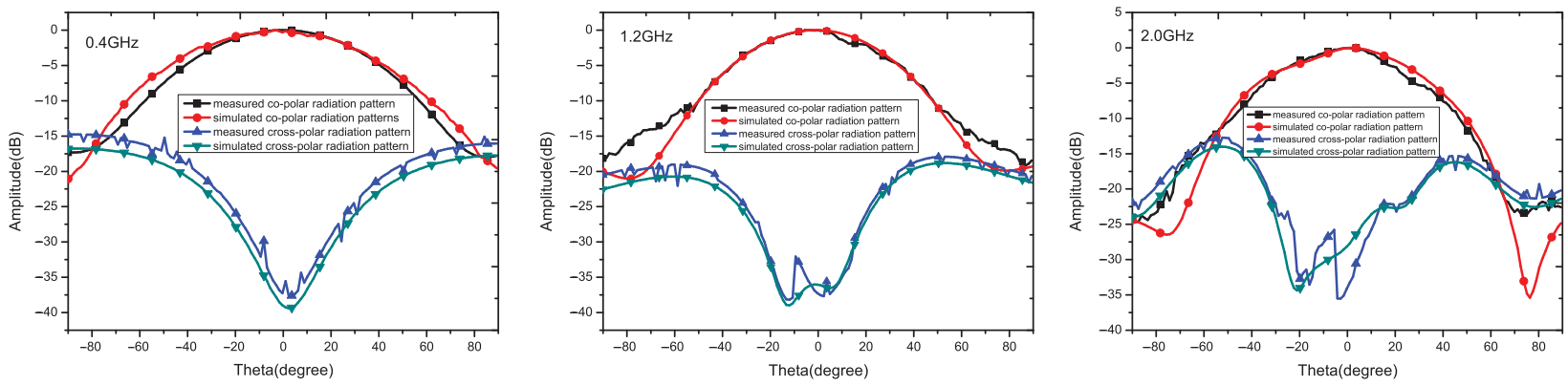

Figure 10. The simulated and measured radiation patterns of the feed. 


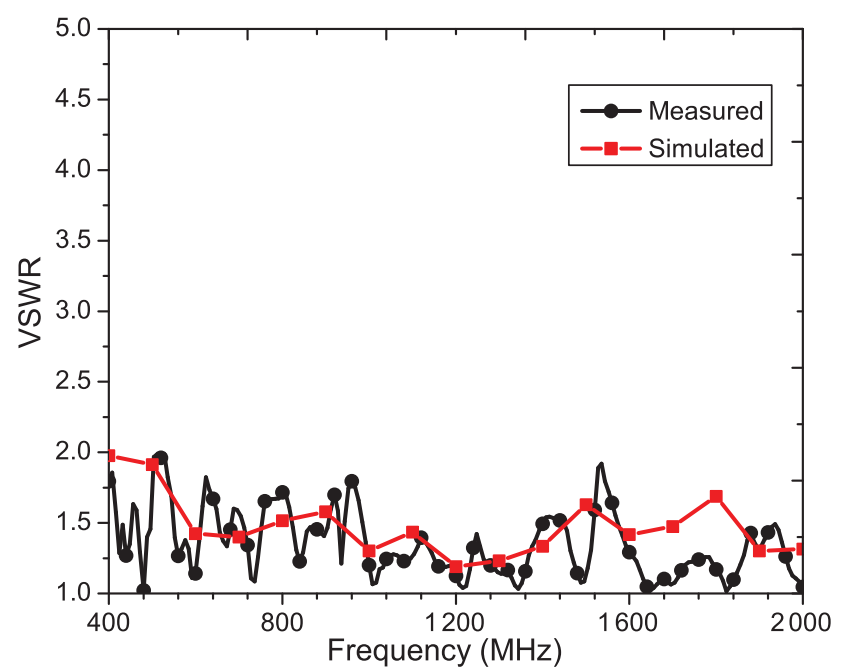

Figure 13. The simulated and measured VSWR.

feed is installed in the focus of the reflector antenna and we use a weather satellite as a radiation source to measure the radiation pattern of this antenna. The antenna uses the satellite beacon signal in azimuth and elevation directions to get the antenna pattern (Yang et al. 2009). Figure 15 shows radiation patterns both in declination and right ascension directions at 1.7 GHz. The first sidelobe of the measured radiations is -14 $\mathrm{dB}$ and the measured pointing and tracking accuracy is 9 arcsec. The measured aperture efficiency is about 0.46 ( $\mathrm{Li}$ et al. 2015a).

\section{CONCLUSION}

In this paper, we demonstrate that a metal ring placed above strip lines can improve the efficiency of the feed significantly. The measurements of the Eleven feed meet the scientific ob-

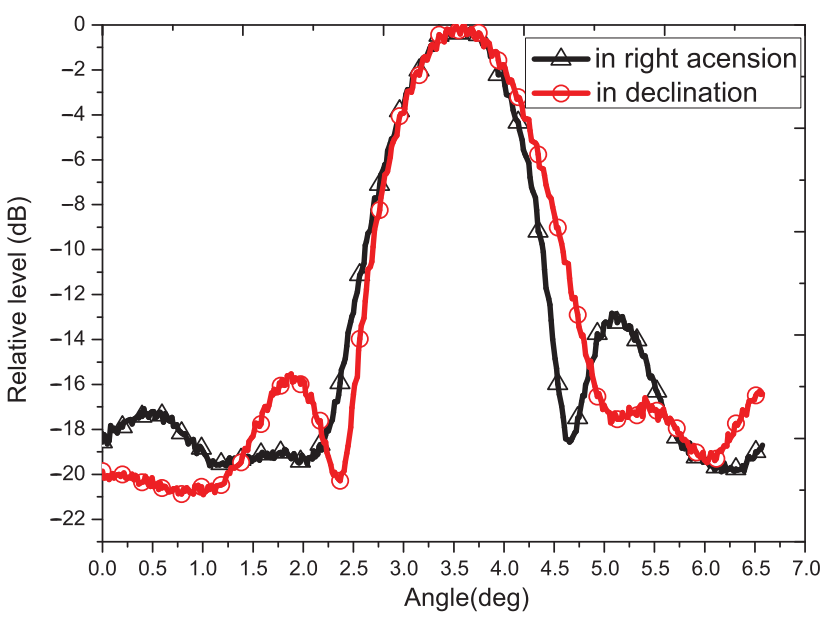

Figure 15. The radiation patterns at $1.7 \mathrm{GHz}$ for both declination and right ascension directions.

jectives of the CSRH programme which requires that the antenna have $\mathrm{VSWR}<2$, axial ratio $<2.5 \mathrm{~dB}$ and $90^{\circ}$ hybrid phase imbalance $<3^{\circ}$ in the ultra-wide frequency band, in order to measure degree of circular polarisation with an accuracy of $10 \%$. The proposed feed design with the inclusion of a metal ring, derived from the traditional designs, may improve the VSWR significantly and this idea can be applied to other feed designs.

\section{ACKNOWLEDGEMENTS}

This work was supported in part by National Major Scientific Equipment Research and Design project under Grant ZDYZ2009-3. The feed measurement was supported by National Natural Science Foundation of China NSFC grants (Nos.: MOST2011CB811401, 11221063, 10778605, 11003028, 11573043, 11433006,61572461, U1231205).
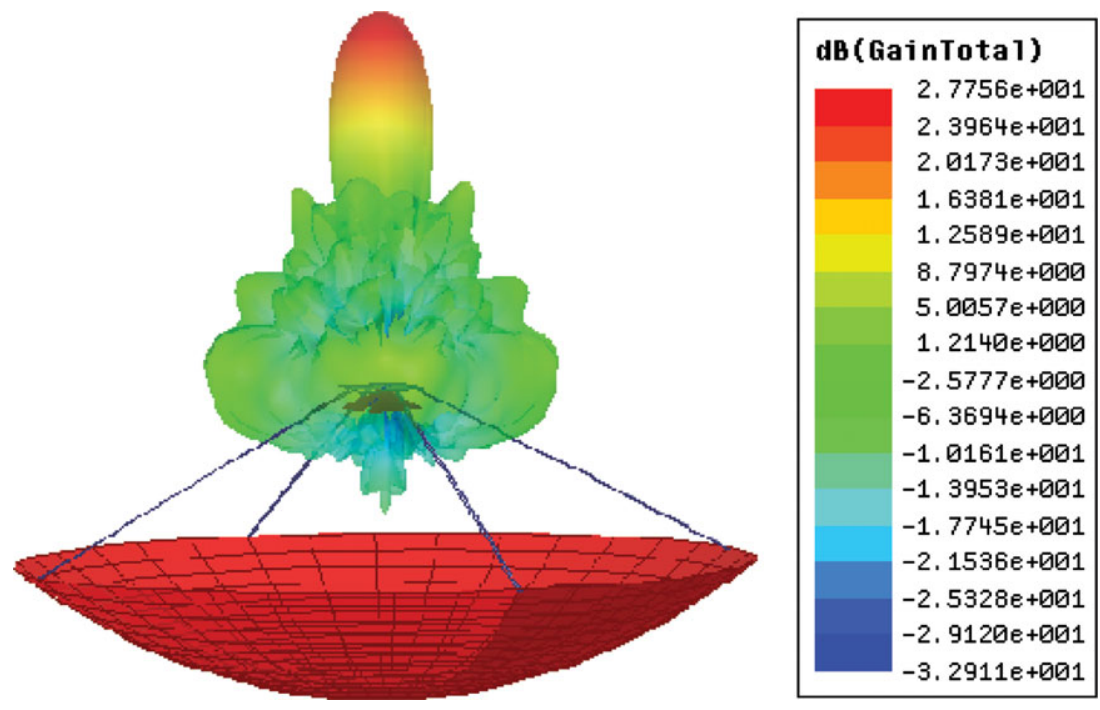

Figure 14. The simulated $3 \mathrm{D}$ radiation patterns at $0.4 \mathrm{GHz}$. 


\section{References}

Acton, L.W. 1966, Magnetodynamic Phenomena in the Solar Atmosphere (Amsterdam: Springer Netherlands)

Aghdam, K. M. P., Farajj-Dana, R., \& Rashed-Mohassel, J. 2005, IEEE Proc. Microw. Antennas Propag., 59, 392

Bennett, J., Anderson, A., McInnes, P., \& Whitaker, A. J. T. 1976, ITAP, 24, 295

Benz, A. O. 2009, 4.1.2.8 Radio Bursts of the Non-Thermal Sun (Berlin Heidelberg: Springer-Verlag)

Richard, C. J., \& Jasik, H. E. 1984, Antenna Engineering Handbook (New York: McGraw-Hill)

Cristal, E., \& Young, L. 1965, ITMTT, 13, 544

Deng, P.-H., Lai, M.-I., Jeng, S.-K., \& Chen, C. H. 2006, ITMTT, 54,4185

Ebenezer, E., Subramanian, K., Ramesh, R., Sundararajan, M., \& Kathiravan, C. 2007, BASI, 35, 111

Kane, S. R., Sawant, H. S., Cecatto, J. R., Andrade, M. C., Fernandes, F. C. R., Karlicky, M., \& Meszarosova, H. 2003, Ad$\mathrm{SpR}, 32,2503$

Kerdraon, A., \& Delouis, J.-M. 1997, Lecture Notes in Physics, 483, 192

Kildal, P.-S. 2000, Foundations of antennas - A unified approach (Sweden: Studentlitteratur), 35

Kim, J. S., Eisenstadt, W., Andrew, M., \& Hanaway, P. 2008, in Microwave Symposium Digest, 2008 IEEE MTT-S International (held in Atlanta, Georgia), 56

Kraus, J., \& Marhefka, R. 2002, Antennas: For All Applications (New York: McGraw-Hill)

Lesovoi, S. V., Altyntsev, A. T., Ivanov, E. F., \& Gubin, A. V. 2014, RAA, 14, 864

Li, S., Yan, Y. H., Chen, Z. J., \& Wang, W. 2014, in General Assembly and Scientific Symposium (URSI GASS), 2014 XXXIth URSI (held in Beijing, China), 1
Li, S., Yan, Y. H., Chen, Z. J., Wang, W., \& Liu, D. H. 2015a, RAA, 15, 1917

Li, S., Yan, Y. H., Chen, Z. J., Wang, W., \& Zhang, F. S. 2015b, PASA, 32

Liu, D., Yan, Y., Zhao, A., \& Wang, W. 2013, AcElS, 41, 570

Nakajima, H., Nishio, M., \& Enome, S. 1994, IEEEP, 82, 705

Olsson, R., Kildal, P. S., \& Weinreb, S. 2006, ITAP, 54, 368

Qing, X., \& Chia, Y. 1999, ElL, 35, 2154

Shelton, J. 1966, ITMTT, 14, 7

Sikina, T. 2010, in IEEE International Symposium on Phased Array Systems and Technology (Piscataway: IEEE), 924

Wang, W., et al. 2013, PASJ, 65, sp18

Xie, Y., Wang, P., Li, L., Zhou, J., \& Zhenya, L. 2007, The Foundmental Analysis of Ansoft HFSS (China: Xidian University), 83

Yan, Y., Zhang, J., Chen, Z., Wang, W., Liu, F., \& Geng, L. 2012, IAUSS, 6

Yan, Y., Zhang, J., Wang, W., Liu, F., Chen, Z., \& Ji, G. 2008, Earth, Moon, and Planets, 104, 97

Yang, J. 2014, in General Assembly and Scientific Symposium (URSI GASS), 2014 XXXIth URSI (held in Beijing, China), 1

Yang, J., Chen, X., Wadefalk, N., \& Kildal, P. S. 2009, IAWPL, 8, 64

Yang, J., \& Kildal, P. S. 2000, ITAP, 48, 524

Yang, J., Pantaleev, M., Billade, B., Ivashina, M., Carozzi, T., Helldner, L., \& Dahlgren, M. 2015, ITAP, 63 , 5955

Yang, J., Pantaleev, M., Kildal, P. S., Klein, B., Karandikar, Y., Helldner, L., Wadefalk, N., \& Beaudoin, C. 2011, ITAP, 59, 1918

Yin, J., Yang, J., Pantaleev, M., \& Helldner, L. 2013, ITAP, 61, 3976

Zhang, H., Peng, Y., \& Xin, H. 2008, IAWPL, 7, 119 